\title{
Highly efficient transfection of human induced pluripotent stem cells using magnetic nanoparticles
}

This article was published in the following Dove Press journal: International Journal of Nanomedicine

\author{
Megan A Yamoah' \\ Maryam Moshref' \\ Janhavi Sharma' \\ Wei Chun Chen' \\ Hannah A Ledford' \\ Jeong Han Lee ${ }^{2}$ \\ Karen S Chavez' \\ Wenying Wang ${ }^{2}$ \\ Javier E López' \\ Deborah K Lieu' \\ Padmini Sirish ${ }^{1,3}$ \\ Xiao-Dong Zhang ${ }^{1,3}$ \\ 'Department of Internal Medicine, \\ University of California, Davis, CA, \\ USA; ${ }^{2}$ Department of Physiology and \\ Cell Biology, University of Nevada, \\ Reno, NV, USA; ' ${ }^{2}$ Department of \\ Veterans Affairs, Northern California \\ Health Care System, Mather, CA, USA
}

Correspondence: Xiao-Dong Zhang Department of Internal Medicine, University of California, 45I E Health Science Drive, Davis, CA 95616, USA $\mathrm{Tel}+\mathrm{I} 5307529717$

Fax +I 5307547167

Email xdzhang@ucdavis.edu

Padmini Sirish

Department of Internal Medicine, University of California, 45I E Health Science Drive, Davis, CA 95616, USA Email psirish@ucdavis.edu
Purpose: The delivery of transgenes into human induced pluripotent stem cell (hiPSC)-derived cardiomyocytes (hiPSC-CMs) represents an important tool in cardiac regeneration with potential for clinical applications. Gene transfection is more difficult, however, for hiPSCs and hiPSC-CMs than for somatic cells. Despite improvements in transfection and transduction, the efficiency, cytotoxicity, safety, and cost of these methods remain unsatisfactory. The objective of this study is to examine gene transfection in hiPSCs and hiPSC-CMs using magnetic nanoparticles (NPs). Methods: Magnetic NPs are unique transfection reagents that form complexes with nucleic acids by ionic interaction. The particles, loaded with nucleic acids, can be guided by a magnetic field to allow their concentration onto the surface of the cell membrane. Subsequent uptake of the loaded particles by the cells allows for high efficiency transfection of the cells with nucleic acids. We developed a new method using magnetic NPs to transfect hiPSCs and hiPSC-CMs. HiPSCs and hiPSC-CMs were cultured and analyzed using confocal microscopy, flow cytometry, and patch clamp recordings to quantify the transfection efficiency and cellular function.

Results: We compared the transfection efficiency of hiPSCs with that of human embryonic kidney (HEK 293) cells. We observed that the average efficiency in hiPSCs was $43 \% \pm 2 \%$ compared to $62 \% \pm 4 \%$ in HEK 293 cells. Further analysis of the transfected hiPSCs showed that the differentiation of hiPSCs to hiPSC-CMs was not altered by NPs. Finally, robust transfection of hiPSC-CMs with an efficiency of $18 \% \pm 2 \%$ was obtained.

Conclusion: The difficult-to-transfect hiPSCs and hiPSC-CMs were efficiently transfected using magnetic NPs. Our study offers a novel approach for transfection of hiPSCs and hiPSCCMs without the need for viral vector generation.

Keywords: human induced pluripotent stem cell-derived cardiomyocytes, therapy, pluripotency, efficiency

\section{Introduction}

Human induced pluripotent stem cells (hiPSCs) are human somatic cells that are genetically reprogrammed into an embryonic-like, pluripotent state capable of differentiating into all three germ layers. Since the original description of induced pluripotent stem cells (iPSCs), ${ }^{1}$ the field has greatly expanded. ${ }^{2-4}$ The hiPSC technology has the potential to revolutionize regenerative and precision medicine by providing differentiated cells for cell-based therapy, disease modeling, drug testing, and high-throughput drug discovery in a patient-specific manner.

A large number of studies have refined the techniques for efficient directeddifferentiation of hiPSCs into cardiomyocytes. ${ }^{5}$ In addition, multiple studies have provided evidence for the application of hiPSC-derived cardiomyocytes (hiPSC-CMs) 
in cardiac transplantation in animal models. ${ }^{6}$ One of the main advantages of utilizing hiPSC-CMs, as opposed to undifferentiated hiPSCs, is the elimination of the risk of teratoma formation. Moreover, hiPSC-CMs serve as an unlimited source of committed human cardiomyocytes.

Genetic modification of hiPSCs represents an essential tool for the study of hiPSCs. Expression of different markers is required in cell-based therapy applications of hiPSCs and hiPSC-CMs. With the advent of genome editing using CRISPR-Cas9 technology, ${ }^{7}$ it has become increasingly feasible to correct disease-causing mutations in patient-specific hiPSCs, in order to create isogenic lines for disease modeling or potential therapeutics. However, there are significant technical challenges for the transfection of hiPSCs and hiPSC-CMs, since these cells are known to be difficult to transfect. ${ }^{8-10}$ Although multiple methods of genetic modifications exist (ie, nucleofection, lipofectamine-mediated transfection, and viral-based transduction), their efficiency, cytotoxicity, safety, and cost remain unsatisfactory. ${ }^{8}$ Increasingly, nanoparticles (NPs) have been used in biomedical research as powerful tools for drug delivery and personalized medicine. ${ }^{11}$ The objective of this study is to examine the efficiency of gene transfection in hiPSCs and hiPSC-CMs using magnetic NPs. Our study offers a novel approach to introduce desired genes into hiPSCs and hiPSC-CMs without the need for viral vector generation.

\section{Materials and methods Cell culture}

HiPSCs (19-9-7T and 6-9-9, WiCell, Madison, WI, USA) were plated in feeder-free conditions using matrigel-coated culture dishes and chemically defined medium, mTeSR ${ }^{\text {TM }} 1$ (Stemcell Technologies, Inc., Cambridge, MA, USA). Cardiac myocytes were generated using a directed differentiation protocol. ${ }^{12}$ Briefly, differentiation of confluent (80\%-90\%) cells was initiated by adding RPMI/B27 medium (Thermo Fisher Scientific, Waltham, MA, USA) lacking insulin and containing the CHIR99021 (Tocris, Minneapolis, MN, USA) for 24 hours, followed by RPMI/B27 media with an inhibitor of Wnt signaling, IWR-1-endo (Tocris). Differentiated hiPSCs were replated on a coverslip prior to transfection and action potential (AP) recordings.

\section{Magnetic-assisted transfection using nanoparticles}

The transfection was conducted following the manufacturer's instructions (Neuromag, OZ Biosciences Inc., San Diego, CA, USA) and published methods. ${ }^{13,14}$ The NPs are positively charged, with a zeta $>+30 \mathrm{mV}$ in water. The size of the NPs ranges from 140 to $200 \mathrm{~nm}$ with the majority around $160 \mathrm{~nm}$, and the particle population is rather homogeneous. Briefly, plasmid DNAs (pIRES2-EGFP, Clontech Laboratories, Inc., Mountain View, CA, USA) or a double fusion construct (an integrating vector) with green fluorescence protein $(\mathrm{GFP})^{15}$ were diluted in cell culture medium, and the NP reagent was added to the culture medium containing DNA. DNA handling followed NIH guidelines. After brief vortexing and 20-minute incubation at room temperature, the medium containing the DNA/nanoparticle complexes was added to the cell culture dish. The dish was then placed on a magnetic plate and incubated in a cell culture incubator for 1,2 , and 4 hours. Cells were harvested or differentiated after 24-48 hours of transfection. For comparison, lipofectamine2000 and -3000 (Thermo Fisher Scientific) were used.

\section{Flow cytometric analysis}

Cells were trypsinized and analyzed for GFP signal using a standard FACScan cytometer (BD Biosciences, San Jose, CA, USA), as we have described. ${ }^{16}$ Briefly, cells were fixed with $0.4 \%$ paraformaldehyde (PFA) before treating with anti-myosin heavy chain antibody (Developmental Studies Hybridoma Bank, Iowa city, IA, USA) in PBS with 5\% donkey serum and $20 \mu \mathrm{g} / \mathrm{mL}$ DNAse-free RNAse (SigmaAldrich Co., St Louis, MO, USA), overnight at $4^{\circ} \mathrm{C}$. Cells were also stained with $40 \mu \mathrm{g} / \mathrm{mL}$ 7-amino-actinomycin D (7AAD, BD Biosciences) to measure the DNA content. Data were collected using a standard FACScan cytometer (BD Biosciences) upgraded to a dual laser system with the addition of a blue laser (15 $\mathrm{mW}$ at $488 \mathrm{~nm}$ ) and a red laser (25 mW at $637 \mathrm{~nm}$ Cytek Development, Inc., Fremont, CA, USA). Data were acquired using CellQuest software (BD Biosciences) and analyzed using FlowJo software (Ver9.4 Treestar Inc., San Carlos, CA, USA). Cells stained with isotype-matched $\mathrm{IgG}$ antibodies were used as controls to determine the positive cell population.

\section{Immunofluorescence confocal microscopy}

Expression of troponin $\mathrm{T}$ in hiPSC-CMs was detected by using mouse monoclonal anti-cardiac troponin $\mathrm{T}$ antibody (Abcam, Burlingame, CA, USA). Images were taken using Zeiss LSM 700 confocal microscope (Carl Zeiss, Oberkochen, Germany).

\section{Electrophysiologic recordings}

Spontaneous action potentials (APs) of hiPSC-CMs were recorded using the perforated-patch recording technique at $35^{\circ} \mathrm{C}$, as we have described. ${ }^{17}$ Briefly, the patch-pipettes 
were backfilled with amphotericin $(200 \mu \mathrm{g} / \mathrm{mL})$. The pipette solution contained (mM) K-glutamate $120, \mathrm{KCl} 25, \mathrm{MgCl}_{2}$ $1, \mathrm{CaCl}_{2} 1$, HEPES ( $N$-2-hydroxyethylpiperazine- $N$ '-2ethanesulphonic acid) 10 , pH 7.4 with $\mathrm{KOH}$. The external solution contained (in $\mathrm{mM}$ ): $\mathrm{NaCl} 138, \mathrm{KCl} 4, \mathrm{MgCl}_{2} 1, \mathrm{CaCl}_{2}$ 2, $\mathrm{NaH}_{2} \mathrm{PO}_{4}$ 0.33, glucose 10, HEPES 10, pH 7.4 with $\mathrm{NaOH}$. The recording was performed using an Axopatch 200A amplifier (Molecular Devices, San Jose, CA, USA). The signal was filtered at $1 \mathrm{kHz}$ using a 4-pole Bessel filter and digitized at sampling frequency of $2 \mathrm{kHz}$. Data analysis was carried out using Clampfit 10 software and graphics software (Origin Lab, Origin 6.0, Northampton, MA, USA).

\section{Statistical analysis}

Data are presented as mean \pm standard error (SE). Statistical comparisons were analyzed by Student's $t$-test or oneway ANOVA followed by Bonferroni tests for post hoc comparison. Statistical significance was considered to be achieved when $P<0.05$, and $\mathrm{n}$ represents the number of independently repeated experiments.

\section{Results \\ Efficient transfection of hiPSCs using magnetic nanoparticles}

NPs have recently been used as powerful tools for drug and gene delivery. ${ }^{11,18}$ Magnetic NPs have been successfully used for transfection of difficult-to-transfect primary neurons. ${ }^{13,14}$ However, the use of magnetic NPs for transfection of hiPSCs remains unknown. We therefore tested the transfection of hiPSCs using magnetic nanoparticles. Figure 1A shows a diagram depicting magnet-assisted transfection (magnetotransfection) using NPs. A schematic representation of the experimental protocol is shown in Figure 1B. We first tested the double fusion construct, and the confocal microscopic images of transfected cells are shown in Figure 1C. Flow cytometric analyses were used to directly quantify nanoparticle-mediated transfection in HEK 293 cells and hiPSCs (Figure 1D and E). We further tested the transfection using a non-integrating GFP construct ( $\mathrm{pIRES2-EGFP)} \mathrm{and} \mathrm{optimized} \mathrm{the} \mathrm{time} \mathrm{needed} \mathrm{for}$ magnetotransfection (Figure $1 \mathrm{~F}$ and $\mathrm{G}$ ). We note a significant increase in percentages of GFP-positive hiPSCs using 4 hours
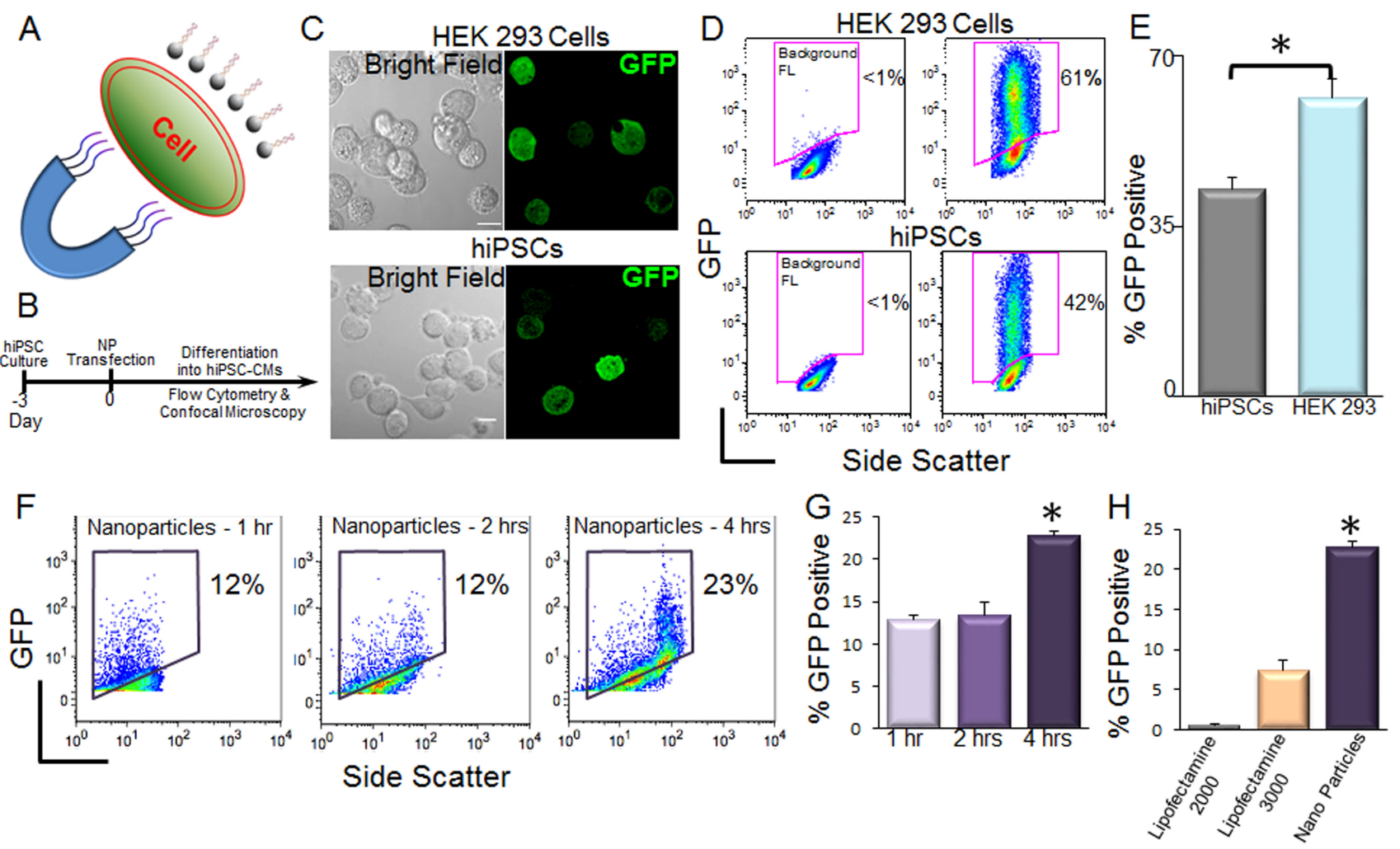

Figure I Transfection of hiPSCs using magnetic nanoparticles.

Notes: (A) Diagram depicting magnetic nanoparticle-mediated transfection. (B) Schematic representation of the experimental protocol. (C) Confocal laser scanning microscopic images of double fusion construct-transfected HEK 293 cells (upper) and hiPSCs (lower). The left panels show the corresponding bright-field images of the cells. Scale bar is $10 \mu \mathrm{m}$. (D) Flow cytometric analyses of transfection efficiencies. Magnetic nanoparticle-treated cells without GFP plasmids were used as control for background fluorescence (Background FL) shown in the left panel. GFP signals were detected from the GFP expression in the cells. (E) Summary data from D (*P<0.05 by Student's $t$-test, $n=3-7$ ). (F) Flow cytometric analysis of pIRES2-EGFP-transfected hiPSCs using I, 2, and 4 hours of magnetotransfection. (G) Summary data from $F(* P<0.05$ by ANOVA, $n=3-7)$. pIRES2-EGFP vector has a lower transfection efficiency compared to double fusion construct (D and $\mathbf{E})$ after 4 hours of magnetotransfection. (H) Comparison of the transfection efficiency of hiPSCs using pIRES2-EGFP vector and lipofectamine-2000, -3000 and nanoparticle-mediated transfections. Four hours of transfection was used for all the conditions $(* P<0.05$ by ANOVA, $n=3-7)$. 
of magnetotransfection. Importantly, there was a significant increase in the efficiency of transfection using magnetotransfection compared to lipofetamine-2000 and -3000 (Figure 1H).

\section{Transfection with NPs did not alter the differentiation of hiPSCs to hiPSC-CMs}

One possible concern using magnetotransfection is whether the procedure may alter the differentiation efficiency of hiPSCs. Here, we directly compared the differentiation efficiency between control (non-transfected) and transfected hiPSCs into cardiomyocytes (CMs). The differentiated CMs exhibited large beating clusters with spontaneous firing APs, consistent with populations of ventricular-like, atrial-like, and nodal-like APs (Figure 2A and B). ${ }^{19}$ There were no significant differences in the efficiency of differentiation into CMs between control hiPSCs and GFP-transfected hiPSCs using NPs (Figure 2C and D).

\section{Magnetotransfection was more efficient than lipofectamine in hiPSC-CMs}

HiPSCs can be differentiated into multiple cell types. HiPSC-CMs have potential for many applications in cardiac regeneration, as well as serving as models for cardiovascular diseases. ${ }^{6,20}$ HiPSC-CMs, as differentiated cells, are even more difficult to transfect compared to hiPSCs. It is critical to further evaluate the efficiency of magnetotransfection in hiPSC-CMs. By using flow cytometric analysis of both myosin heavy chain (MyHC) and GFP positive cells, we directly demonstrated that magnetotransfection was more efficient than lipofectamine not only in hiPSCs, but also in hiPSCCMs (Figure 2E and F) using pIRES2-EGFP construct.

\section{Discussion}

Since the original description of iPSCs, ${ }^{1}$ the wide-reaching potentials of the technology have been rapidly realized for
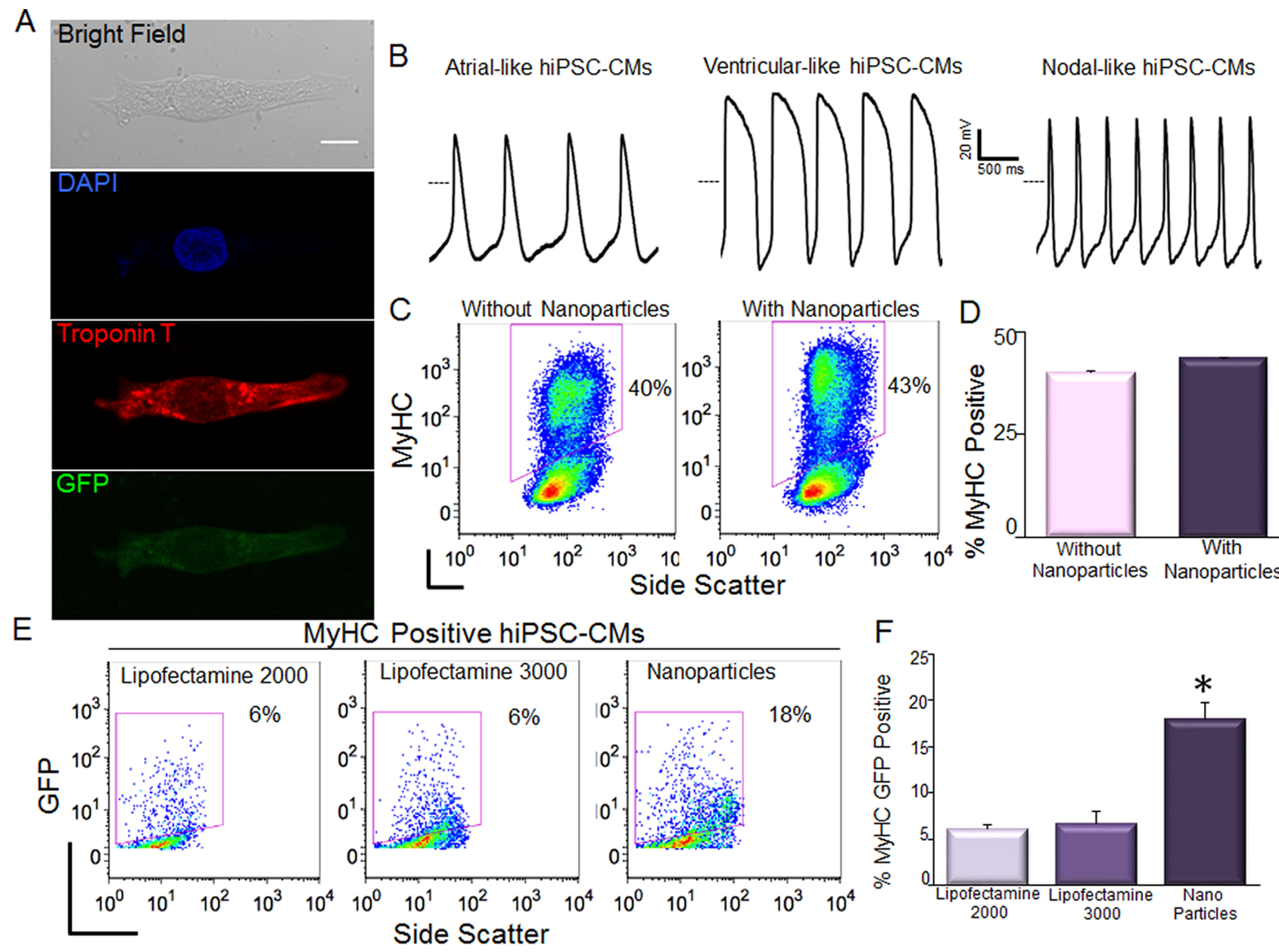

Nodal-like hiPSC-CMs

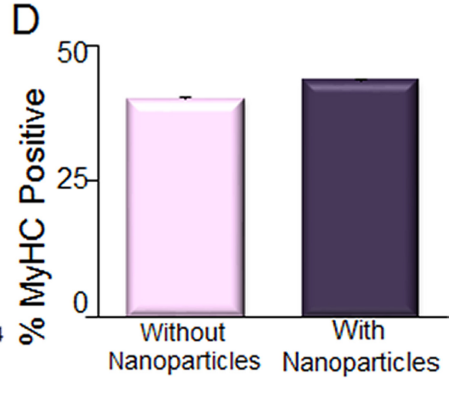

E

MyHC Positive hiPSC-CMs

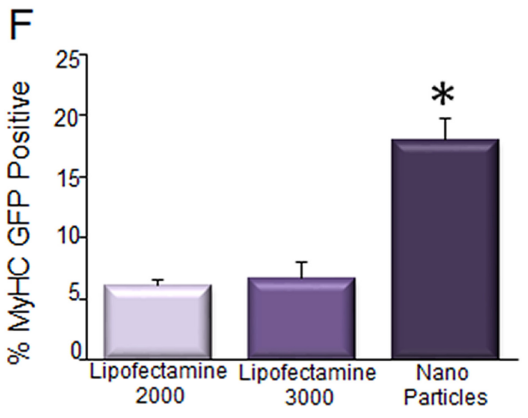

Figure 2 Transfection of hiPSC-CMs using magnetic nanoparticles.

Notes: (A) Confocal laser scanning microscopic images of GFP-transfected hiPSC-CM. Scale bar is $10 \mu \mathrm{m}$. (B) hiPSC-CMs exhibit spontaneous APs with ventricular-like, atrial-like, and nodal-like characteristics. The dotted line represents $0 \mathrm{mV}$. (C, D) Assessment of the efficiency of differentiation into CMs in control hiPSCs compared to hiPSCs transfected with double fusion construct using nanoparticles by analysis of myosin heavy chain (MyHC) positive cells. Summary data are shown in the right panels. (E, F) Comparison of the transfection efficiency in double positive hiPSC-CMs (MyHC + GFP $)$ using pIRES2-EGFP vector and lipofectamine-2000, -3000, and nanoparticlemediated transfections. Data were collected 4 hours after transfection $(* P<0.05$ by ANOVA, $n=3)$. 
both regenerative and precision medicine. The utilization of hiPSCs enables the development of an unlimited source of any human cell types needed for therapeutic and precision medicine. Genetic modification and expression of different reporters are essential for studies to evaluate cell-based therapy applications of hiPSCs and hiPSC-CMs. However, significant technical challenges exist for the transgene delivery into hiPSCs. ${ }^{8-10}$ Several methods have been developed for transgene delivery, which are mainly catogorized into viral and non-viral methods. ${ }^{8-10}$ Three types of viral vectors are widely used in the transgene delivery, including adenoviral, lentiviral, and adeno-associated viral vectors. The advantage of viral vectors lies in their high delivery efficacy, but their use also raises safety concerns including the cytotoxicity, cellular immune responses, and transgene integration into host genome. Non-viral methods, including mechanical and electrical methods such as injection or electroporation, and chemical methods such as lipofection, are potentially safer alternatives for transgene delivery into iPSCs, but the transfection efficiency is relatively lower., ${ }^{9,10}$ For iPSC-CMs and embryonic stem cell-derived cardomyocytes (ESC-CMs), viral transduction and nucleofection are commonly used for transgene delivery. ${ }^{21-24}$

Magnetic NPs have been used for the transfection of cell lines and primary cells including neurons. ${ }^{13,14,25}$ Magnetic nanoparticle-mediated gene transfer offers significant advantages over other gene transfer methods, such as high efficiency, low cytotoxicity, low cost, directional and distal controllability, efficient in vivo applications, and lack of immune responses. ${ }^{25-28}$ Recently, magnetic NPs have been used for gene delivery to neural precursor/stem cells. ${ }^{26,27,29}$ However, whether magnetic nanoparticles can be efficiently used for transfection of iPSCs and iPSC-CMs has not been addressed and reported. Here, we demonstrate that the difficult-to-transfect hiPSCs and hiPSC-CMs can be efficiently transfected using magnetic NPs.

Our study offers a novel approach to introduce transgenes into hiPSCs and hiPSC-CMs without the need for viral vector generation. Indeed, our findings transcend the benefits for hiPSCs and hiPSC-CMs. The technique may represent a non-viral method for the generation of hiPSCs and thus avoid the risk of genomic insertions inherent in some integrating viral methods. The potential application will be the genetic modification of hiPSCs and hiPSC-CMs for in vitro guided differentiation and in vivo transplantation for tissue regeneration and repair. The distal control of nanoparticles by a magnetic field will further potentiate the in vivo application and delivery of nucleic acids to specific organs for targeted gene therapy. The possible limitation may come from the cytotoxicity of magnetic NPs, which results from the accumulation of NPs in endosomes and/or vacuoles in cells. However, NPs will be degraded through normal iron metabolism over time, although the mechanism is still not well understood. ${ }^{11,25}$ For future studies, transfection efficiencies between different types of constructs need to be addressed.

\section{Acknowledgments}

We thank Dr Joseph Wu (Stanford University) for providing the double fusion construct, and Dr Nipavan Chiamvimonvat for her constructive advice on the study. This work was supported by the National Heart, Lung, and Blood Institute of the National Institutes of Health (NIH) (grant number R56HL138392 to XDZ), American Heart Association (AHA) (grant number 14BGIA18870087 to XDZ), NIH K12 (grant number UL1 TR000002 to JEL) and Harold Amos Medical Faculty Development Program, RWJ Foundation (to JEL), California Institute of Regenerative Medicine (CIRM) (grant number RB4-05764 to DKL), Postdoctoral Fellowship Awards to PS and JS from CIRM Stem Cell Training Program to UC Davis (grant number TG2-01163), NIH NRSA F31 Predoctoral Award (F31 HL136120 to HAL), Predoctoral (HAL) and Postdoctoral (PS) Fellowship Award from NIH T32 Training Grant in Basic and Translational Cardiovascular Science (grant number T32 HL08350), AHA Postdoctoral Research Award (grant number 16POST26970007 to PS), AHA Career Development Award (grant number 18CDA34110060 to PS), and Harold S. Geneen Charitable Trust Award (PS). The content is solely the responsibility of the authors and does not necessarily represent the official views of the NIH.

\section{Author contributions}

Zhang XD developed the concept and designed the study, performed cell transfection, confocal imaging and patch clamp recordings, made figures, wrote and revised the manuscript; Sirish $\mathrm{P}$ helped to design the study and performed the hiPSC and hiPSC-CM culture and flow cytometric analysis, made figures, and revised the manuscript; Yamoah MA helped to design the study, performed the major magnetic transfection experiments, confocal imaging and part of flow cytometric analysis, and wrote and revised the manuscript; Maryam Moshref performed part of the magnetic transfection experiments, conducted immunostaining and confocal imaging; Sharma J performed part of the hiPSC-CM culture; Chen WC helped on patch clamp recordings and cell line culture; Ledford HA helped on the cell line culture and lipofactamine transfection, and revised the manuscript; Lee $\mathrm{JH}$ helped on the confocal imaging; Chavez KS helped on 
the hiPSC-CM culture; Wang W helped on the improvement of magnetic transfection protocol; López JE helped on the improvement of hiPSC and hiPSC-CM culture protocols, and revised the manuscript; Lieu DK helped on the improvement of hiPSC and hiPSC-CM culture protocols. All authors contributed to data analysis, drafting and revising the article, gave final approval of the version to be published, and agree to be accountable for all aspects of the work.

\section{Disclosure}

Deborah Lieu reports personal fees from Novoheart Ltd, outside the submitted work. The authors report no other conflicts of interest in this work.

\section{References}

1. Takahashi K, Yamanaka S. Induction of pluripotent stem cells from mouse embryonic and adult fibroblast cultures by defined factors. Cell. 2006;126(4):663-676.

2. Yu J, Vodyanik MA, Smuga-Otto K, et al. Induced pluripotent stem cell lines derived from human somatic cells. Science. 2007;318(5858): 1917-1920.

3. Takahashi K, Tanabe K, Ohnuki M, et al. Induction of pluripotent stem cells from adult human fibroblasts by defined factors. Cell. 2007; 131(5):861-872.

4. Shi Y, Inoue H, Wu JC, Yamanaka S. Induced pluripotent stem cell technology: a decade of progress. Nat Rev Drug Discov. 2017;16(2): 115-130.

5. Lian X, Hsiao C, Wilson G, et al. Robust cardiomyocyte differentiation from human pluripotent stem cells via temporal modulation of canonical Wnt signaling. Proc Natl Acad Sci U S A. 2012;109(27): E1848-E1857.

6. Lalit PA, Hei DJ, Raval AN, Kamp TJ. Induced pluripotent stem cells for post-myocardial infarction repair: remarkable opportunities and challenges. Circ Res. 2014;114(8):1328-1345.

7. Cong L, Ran FA, Cox D, et al. Multiplex genome engineering using CRISPR/Cas systems. Science. 2013;339(6121):819-823.

8. Chatterjee P, Cheung Y, Liew C. Transfecting and nucleofecting human induced pluripotent stem cells. J Vis Exp. 2011 (56):3110.

9. Fontes A, Lakshmipathy U. Advances in genetic modification of pluripotent stem cells. Biotechnol Adv. 2013;31(7):994-1001.

10. Rapti K, Stillitano F, Karakikes I, et al. Effectiveness of gene delivery systems for pluripotent and differentiated cells. Mol Ther Methods Clin Dev. 2015;2:14067.

11. Zhang L, Gu FX, Chan JM, Wang AZ, Langer RS, Farokhzad OC. Nanoparticles in medicine: therapeutic applications and developments. Clin Pharmacol Ther. 2008;83(5):761-769.

12. Lian X, Zhang J, Azarin SM, et al. Directed cardiomyocyte differentiation from human pluripotent stem cells by modulating Wnt/ $\beta$-catenin signaling under fully defined conditions. Nat Protoc. 2013;8(1):162-175.
13. Underhill SM, Wheeler DS, Li M, Watts SD, Ingram SL, Amara SG. Amphetamine modulates excitatory neurotransmission through endocytosis of the glutamate transporter EAAT3 in dopamine neurons. Neuron. 2014;83(2):404-416.

14. Wang R, Palavicini JP, Wang H, et al. RanBP9 overexpression accelerates loss of dendritic spines in a mouse model of Alzheimer's disease. Neurobiol Dis. 2014;69:169-179.

15. Lee AS, Wu JC, Jc W. Imaging of embryonic stem cell migration in vivo. Methods Mol Biol. 2011;750:101-114.

16. Sirish P, Li N, Timofeyev V, et al. Molecular Mechanisms and New Treatment Paradigm for Atrial Fibrillation. Circ Arrhythm Electrophysiol. 2016;9(5):e003721.

17. Zhang XD, Timofeyev V, Li N, et al. Critical roles of a small conductance $\mathrm{Ca}^{2+}$-activated $\mathrm{K}^{+}$channel (SK3) in the repolarization process of atrial myocytes. Cardiovasc Res. 2014;101(2):317-325.

18. Chou LY, Ming K, Chan WC. Strategies for the intracellular delivery of nanoparticles. Chem Soc Rev. 2011;40(1):233-245.

19. Zhang J, Wilson GF, Soerens AG, et al. Functional cardiomyocytes derived from human induced pluripotent stem cells. Circ Res. 2009; 104(4):e30-e41.

20. Doyle MJ, Lohr JL, Chapman CS, Koyano-Nakagawa N, Garry MG, Garry DJ. Human Induced Pluripotent Stem Cell-Derived Cardiomyocytes as a Model for Heart Development and Congenital Heart Disease. Stem Cell Rev. 2015;11(5):710-727.

21. Lieu DK, Fu JD, Chiamvimonvat N, et al. Mechanism-based facilitated maturation of human pluripotent stem cell-derived cardiomyocytes. Circ Arrhythm Electrophysiol. 2013;6(1):191-201.

22. Saito Y, Nakamura K, Yoshida M, et al. Enhancement of Spontaneous Activity by HCN4 Overexpression in Mouse Embryonic Stem CellDerived Cardiomyocytes - A Possible Biological Pacemaker. PLoS One. 2015;10(9):e0138193.

23. Schweizer PA, Darche FF, Ullrich ND, et al. Subtype-specific differentiation of cardiac pacemaker cell clusters from human induced pluripotent stem cells. Stem Cell Res Ther. 2017;8(1):229.

24. Stillitano F, Karakikes I, Hajjar RJ. Gene Transfer in Cardiomyocytes Derived from ES and iPS Cells. Methods Mol Biol. 2017;1521: 183-193.

25. Plank C, Zelphati O, Mykhaylyk O. Magnetically enhanced nucleic acid delivery. Ten years of magnetofection-progress and prospects. Adv Drug Deliv Rev. 2011;63(14-15):1300-1331.

26. Pickard MR, Barraud P, Chari DM. The transfection of multipotent neural precursor/stem cell transplant populations with magnetic nanoparticles. Biomaterials. 2011;32(9):2274-2284.

27. Adams CF, Pickard MR, Chari DM. Magnetic nanoparticle mediated transfection of neural stem cell suspension cultures is enhanced by applied oscillating magnetic fields. Nanomedicine. 2013;9(6):737-741.

28. Corchero JL, Villaverde A. Biomedical applications of distally controlled magnetic nanoparticles. Trends Biotechnol. 2009;27(8):468-476.

29. Pickard MR, Adams CF, Chari DM. Magnetic Nanoparticle-Mediated Gene Delivery to Two- and Three-Dimensional Neural Stem Cell Cultures: Magnet-Assisted Transfection and Multifection Approaches to Enhance Outcomes. Curr Protoc Stem Cell Biol. 2017;40:2D 19.12D.19.16.
International Journal of Nanomedicine

\section{Publish your work in this journal}

The International Journal of Nanomedicine is an international, peerreviewed journal focusing on the application of nanotechnology in diagnostics, therapeutics, and drug delivery systems throughout the biomedical field. This journal is indexed on PubMed Central, MedLine, CAS, SciSearch ${ }^{\circledR}$, Current Contents ${ }^{\circledR} /$ Clinical Medicine,

\section{Dovepress}

Journal Citation Reports/Science Edition, EMBase, Scopus and the Elsevier Bibliographic databases. The manuscript management system is completely online and includes a very quick and fair peer-review system, which is all easy to use. Visit http://www.dovepress.com/ testimonials.php to read real quotes from published authors. 